\title{
Editorial
}

\section{Ultrasound in Regional Anesthesia: How far have We progressed?}

Ultrasound (US) in regional anesthesia has helped us to move from loss of resistance, blind infiltration and paresthesia techniques to more precise placement of local anesthetic (LA) in the desired anatomical area. Its advantages are: noninvasive, safe, simple, real-time images, no direct adverse effects like radiation to the patient or proceduralist. The landmark techniques practiced for decades, which are still used and important. How far have we come from its initial use today? Historically, the first Doppler flow ultrasound-guided supraclavicular block was performed by La Grange

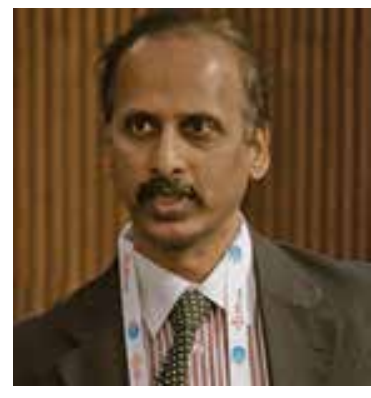
et al in 1978. ${ }^{1}$ It was indirectly used with the main limitation being poor resolution. The technology was so slow in those days that it took more than 15 years to report direct ultrasound-guided supraclavicular brachial plexus block by Kapral in $1994 .^{2}$ There have been various issues like expensive ultrasound machine and lack of knowledge of technology, training and awareness of its use in other regional block techniques. At that time very few people had the enthusiasm and vision to improve. Among the few, the authors like Grau and Chan contributed a lot on improving US for nerve blocks.,

In the last two decades, more interest was generated by the anesthesiologists across the globe. Many scientists have dedicated their software work and research to improve high resolution US visualization techniques, making various frequency transducers, color Doppler for neuroaxial and deeper blocks. Due to industrial interest, affordable and portable US machines were available and this leads to US culture among anesthetists and pain doctors. Industries did contribute designing technology to perform diagnostic, therapeutic, imaging and intervention procedures with a strong focus on US. Now, we can perform most of the regional blocks under US. There are numerous journals dedicated to US and regional anesthesia including validated certification for practice.

We are in the era of evidence-based medicine, why US should replace traditional techniques? Is US safe and superior to landmark techniques or nerve stimulator technique? Recent review by Perlas showed that US is favorable. ${ }^{5}$

In peripheral nerve blocks, it minimizes the chance of pneumothorax, intravascular or intraneural injection. With US, you can see the tip of the needle avoiding multiple passes and the precise site of LA drug spread, reducing the volume of the drug. One study including a systemic review favored its use in difficult spinal and epidural anesthesia. ${ }^{6,7}$ It predicts intervertebral spaces with greater precision than palpation and estimates the depth of epidural space and angle of needle insertion. Even experienced anesthetist may have neuroaxial block difficulties in situations like obesity or scoliosis, and US has been proved to prevent multiple needle passes and vascular or dural puncture. Apart from the advantage of success, will it reduce the complications? Apparently, it is not clear. According to Auroy et al, most of the complications were drug-related and not due to technique. ${ }^{8}$ But in this review article, evidence is in favor of US, specifically when performing a neuroaxial block. ${ }^{5}$ Along with this fascinated technology, we come across new problems like the gel use and infections. There is need to use only sterile gel. There were reports of gel introduced in to the tissues. ${ }^{9}$

I remember doing blind transversus abdominis plane (TAP) block and other nerve blocks when US machines were not available in district hospital in India. It requires hospital administrators to be convinced, there will be savings to the institute in performing safe and effective procedures and reduced complications, part of hospital clinical indicators. It is not only the availability of technology but also training and willingness to learn and administrators, teachers in medical schools to promote its use effectively. Hospital spends on US will pay dividends by its precise use and avoid preventable complications related to unintended structures penetration or LA toxicity.

We do not have conclusive randomized controlled trial study that US is absolutely better than traditional technique. We can still improve enhancement of needle visualization. Though three-dimensional (3D) image technology has arrived, we still find visualization of complex bony structures difficult. Cost and training are still a barrier to use in daily clinical practice. We still need affordable US machines available to the developing world's regional anesthetic practitioners which should be safe and cost-effective in increasing work efficiency. 


\section{REFERENCES}

1. La Grange P, Foster PA, Pretorius LK. Application of the Doppler ultrasound blood flow detector in supraclavicular brachial plexus block. Br J Anaesth 1978;50(10):965-967.

2. KapralS, Kraft P, Eibenberger K, et al. Ultrasound-guided supraclavicular approach for regional anesthesia of the brachial plexus. Anesth Analg 1994;78(3):507-513.

3. Grau T. Ultrasonography in the current practice of regional anaesthesia. Best Pract Res Clin Anaesthesiol 2005 Jun;19(2):175-200.

4. Chan VWS. The use of ultrasound for peripheral nerve blocks. In: Boezaart AP, editor Anaesthesia and Orthopaedic Surgery. New York: McGraw hill; 2006. p. 283-290.

5. Perlas A. Evidence for the use of ultrasound in neuraxial blocks: review article. Regional Anesthesia and Pain Medicine 2010;35(suppl 2): S43-S46.

6. Ki JC, Perlas A, Chan V, Brown-Shreves D, Koshkin A, Vaishnav V. Ultrasound imaging facilitates spinal anaesthesia in adults with difficult surface landmarks. Anaesthesilogy 2011;115(1):94-101.

7. Shaikh F, Brzezinski J, Alexander S, Arzola C, Carvalho JCA, Beyene J, Sung L. Ultrasound imaging for lumbar puncture and epidural catheterisation: systemic review and metanalysis. BMJ 2013;346(3):f1720.

8. Auroy Y, Benhamou D, Bargues L, Ecoffey C, Falissard B, Mercier FJ, Bouaziz H, Samii K. Major complications of regional anesthesia in France. Anesthesiology 2002;97(5):1274-1280.

9. Belavy D. Regional anesthesia needles can introduce ultrasound gel into tissues. Anesthesia and Analgesia 2010;111(3):811-812.

Vasanth Rao Kadam

MBBS, MD (Anesthesiology), DNB, MNAMS, FANZCA

Supervisor (Module), Postgraduate Training SA

ANZ College of Anaesthetists (2006-2012)

Senior Consultant, The University of Adelaide

Adelaide, Australia 